\title{
Calcium Accumulation in Visual Interneurons of the Fly: Stimulus Dependence and Relationship to Membrane Potential
}

\author{
MARTIN EGELHAAF AND ALEXANDER BORST \\ Max-Planck-Institut für biologische Kybernetik, D-72076 Tübingen, Germany
}

\section{SUMMARY AND CONCLUSIONS}

1. The large motion-sensitive tangential neurons in the fly third visual neuropil spatially pool the postsynaptic signals of many local elements. The changes in membrane potential and calcium concentration induced in these cells by visual motion are analyzed in vivo by simultaneous optical and intracellular voltage recording techniques.

2. Visual motion in the preferred direction leads to depolarization of the cell and to calcium accumulation mainly in the axon terminal, the soma, and the dendritic tree. During motion in the null direction, the cell hyperpolarizes and virtually no changes in calcium concentration can be observed.

3. Dendritic calcium accumulation is first restricted to those dendritic branches that are close to the sites of direct synaptic input. In other parts of the dendrite the calcium concentration increases more slowly and usually reaches only lower levels.

4. Calcium starts accumulating at the onset of motion. However, the calcium concentration reaches its final steady-state level much later than the corresponding membrane potential changes. Even if these are completely transient at high temporal frequencies of pattern motion, the calcium signal stays high until the stimulus pattern stops moving.

5. The amplitude of the calcium signal depends on the temporal frequency of pattern motion in a similar way as do the corresponding membrane potential changes. However, there exist differences that can be attributed to the different time courses of both signals.

6. Depolarization of the dendritic tree by current injection through a microelectrode leads to similar changes in calcium accumulation as does activation by synaptic input, suggesting that calcium enters the cell via voltage-dependent channels. The possible function of calcium channels for dendritic integration of synaptic input is discussed.

\section{INTRODUCTION}

Despite their vast anatomic diversity, neurons are often conceived as quite simple and uniform processing units. According to this view, the main function of the dendritic tree is to summate the synaptic input signals and to transform them into a sequence of action potentials. Only recently has it been appreciated that dendrites may be much more than passive summators of spatially distributed synaptic input signals. Rather, there is continually increasing experimental evidence that dendrites house a large number of voltagedependent and second-messenger-gated ion channels (Llinás 1988), rendering nerve cells much more complex computational devices than previously thought. Examples of cells where such active dendritic membrane properties are being investigated in particular detail are the Purkinje cells of the cerebellum (Chan et al. 1989; Lev-Ram et al. 1992; Llinás and Sugimori 1980a,b; Midtgaard 1994; Miyakawa et al.
1992), the hippocampal pyramid cells (Jaffe et al. 1992; Miyakawa et al. 1992; Regehr and Tank 1990, 1992; Regehr et al. 1989), and nonspiking local interneurons in the motor system of the locust (Laurent 1990, 1993). However, despite the great knowledge accumulated at the biophysical level, the computational tasks of these cells are often not yet sufficiently understood. The significance of all the active dendritic processes for the computations being performed by the neurons has been clarified in only a few cases (Laurent 1990, 1993; Sobel and Tank 1994).

A group of visual interneurons of the fly, the so-called tangential cells, lend themselves particularly well to an analysis of how the information processing capabilities of nerve cells are linked to the underlying dendritic mechanisms. This is because on the one hand, a specific function can be attributed to the tangential cells in visual orientation behavior of the fly, and on the other hand, these cells are well accessible to electrophysiological and optical recording techniques both in vivo and in vitro. The tangential cells are situated in the posterior part of the third visual neuropil, the lobula plate, and comprise a group of 50-60 cells that are all uniquely identifiable on the basis of their anatomy and response characteristics (Hausen 1981; Hausen and Egelhaaf 1989). With their extended dendritic trees, the tangential cells collect the output signals of many retinotopically arranged local motion-sensitive elements and thus respond to visual motion in a directionally selective manner. The cellular nature of these local input elements can only be tentatively assessed on the basis of anatomic, electrophysiological, and deoxyglucose analyses (Bausenwein et al. 1992; Gilbert et al. 1991; Strausfeld and Lee 1991). However, the functional properties of the local input elements could be derived from the properties of the tangential cells and can be described in great detail by the formal correlation detector model (Borst and Egelhaaf 1989, 1993; Egelhaaf and Borst 1993a; Reichardt 1961, 1987). According to this model, the light intensity measured at one location in visual space is low-pass filtered and subsequently correlated with the instantaneous light intensity derived from a neighboring location. This operation is performed for any location in visual space in several orientations, resulting in a retinotopic map of visual motion signals for different directions of motion. Local motion detectors tuned to opposite directions are thought to impinge on the dendrites of the tangential cells with excitatory and inhibitory synapses, respectively, leading to an enhanced direction selectivity in the latter (Borst and Egelhaaf 1990; Egelhaaf et al. 1989). In electrophysiological, behavioral, and ablation studies, some tangential cells were shown to control specific components 
of visual orientation such as compensatory optomotor course stabilization or figure-ground segregation (Egelhaaf and Borst 1993b; Egelhaaf et al. 1988; Hausen and Egelhaaf 1989).

In the present account we lay our emphasis on a particular group of tangential cells, the so-called horizontal-motionsensitive (HS) and vertical-motion-sensitive (VS) cells. Both cell classes are activated by motion in a given direction and inhibited by motion in the respective reverse direction. The HS cells comprise three cells with their receptive fields in the dorsal (HSN), medial (HSE), or ventral part of the visual field of the ipsilateral eye. They are all sensitive to horizontal motion from the front to the back (their "preferred direction") and are inhibited by motion in the opposite direction (their "null direction") (Hausen 1982a,b). The VS cells comprise 11 different cells, named sequentially VS1-VS11, with receptive fields located in the frontal (VS1) to increasingly more lateral parts of the ipsilateral eye (Hengstenberg et al. 1982). Roughly speaking, the VS cells are most sensitive to downward motion (Hengstenberg 1982), although recent detailed analyses hint at a more refined structure of their receptive fields (Krapp et al. 1994). Both HS and VS cells respond to motion mainly with graded changes in membrane potential, even when recorded from near their axon terminals. These graded membrane potential changes are superimposed by small-amplitude spikelike depolarizations (Haag et al. 1993; Hausen 1982b; Hengstenberg 1977). To study the mechanisms underlying dendritic integration in these neurons, we adapted the optical recording technique to the visual system of the fly, which allowed us to monitor the spatial distribution of various ions in the dendritic tree during visual motion stimulation. This proved to be possible because the dendritic trees of the tangential cells arborize in an almost two-dimensional layer directly below the surface of the brain. A previous study (Borst and Egelhaaf 1992) revealed that on visual stimulation, calcium accumulates in the dendrites of the tangential cells. In the present study we further analyze the nature of the calcium signal by measuring the spatiotemporal calcium concentration changes during localized or global activation of the cell by visual stimulation as well as by current injection into the dendrite, and by relating these concentration changes to the simultaneously recorded membrane potential changes.

\section{METHODS}

\section{Preparation}

Experiments were performed with 1- to 2-day-old female blowflies (Calliphora erythrocephala). The animals were anesthesized with carbon dioxide and mounted ventral side up with wax on a small piece of glass. The legs were amputated. The head capsule was opened from behind, and the trachea and air sacs were removed to gain access to the posterior part of the third visual neuropil, the lobula plate. To avoid desiccation of the brain, the head capsule was supplied with Ringer solution (for a formula see Hausen 1982a ). Movements of the brain caused by peristaltic contractions of the esophagus and by pumping movements of the abdomen were prevented by cutting away the proboscis and the rear half of the abdomen and pulling out the gut. All wounds were sealed with wax.

\section{Optical recording and electrophysiology}

The fly was mounted under an upright microscope (Zeiss Universal) on a platform that could be moved in all three directions. To allow for focusing on the injected cell while it was impaled with the microelectrode, the micromanipulators for the different and indifferent electrodes were also fixed to this platform. The preparation was viewed with a Zeiss UD20 long-distance objective (numerical aperture 0.56 ). The cells were injected with either a 12$\mathrm{mM}$ solution of the potassium salt of fura-2 (Molecular Probes) in $500 \mathrm{mM} \mathrm{KCl}$ or a $9-\mathrm{mM}$ solution of the potassium salt of Calcium Green (Molecular Probes) in $200 \mathrm{mM}$ potassium acetate. Electrodes were pulled on a Brown-Flaming puller (Sutter Instruments) from 1.0-mm tubing (Clark Electromedical GC100) and had resistances of $\sim 50 \mathrm{M} \Omega$. The indifferent electrode was used for supply of Ringer solution. Electrophysiological signals were stored and evaluated on a PC (IBM compatible 80486) through an A-D converter (DT2801A, Data Translation) at $1 \mathrm{kHz}$. The software was written in ASYST (Keithley) and ensured synchronization with the image acquisition. For optical recording, the preparation was illuminated with an epifluorescence light source fitted with an appropriate filter combination. (For fura imaging: BP 380$\mathrm{nm}$ or $349-\mathrm{nm}$ excitation filters, bandwidth $10 \mathrm{~nm}$; a $410-\mathrm{nm}$ dichroic mirror, and a BP 500- to 530-nm barrier filter. For Calcium Green imaging: BP 472-nm excitation filter, bandwidth $17 \mathrm{~nm}$; a 510-nm dichroic mirror, and a BP 520- to $560-\mathrm{nm}$ barrier filter.) The imaging system (Photometrics) consisted of a Peltier-cooled camera head (CH 250A) with a charge-coupled device chip (PM512; ultralow dark current: $512 \times 512$ pixels), an electronic unit (CE 200A) equipped with a 50-kHz, 16-bit A-D converter, and a controller board (NU 200). Images were acquired and evaluated by a software package (IPLab, Signal Analytics, Vienna, VA) on a Macintosh IIfx computer (Apple). For a comparison between the onset of the calcium signal and the membrane potential at a high temporal resolution, the charge-coupled-device (CCD) camera was replaced in some experiments by a single photodiode (sensitivity range $350-550 \mathrm{~nm}$; Centronic OSD50-5).

\section{Experimental procedure}

The dye (fura- 2 or Calcium Green, Molecular Probes) was injected into the cell $(10-30 \mathrm{~min} ;-1$ to $-3 \mathrm{nA})$ until the major cellular processes could be clearly discerned from the background. Then the dye was allowed to diffuse throughout the cell for 10-30 min. However, the most distal dendritic branches on which most of the input synapses of these cells reside (Hausen et al. 1980) could not be resolved in the fluorescence images in vivo, as is suggested by a comparison with anatomic data using convential dyes and sectioned material (Hausen 1982a; Hausen et al. 1980; Hengstenberg et al. 1982). At rest, the fluorescence of the main cellular branches was $\geq 2-5$ times higher than that of the background. In part of the experiments, the electrical activity of the cell was simultaneously recorded to allow comparison with the optical recording data. During the experiments a sequence of pictures of the cell was taken at 1.5-s intervals under epifluorescence illumination with the approriate filter combination. The exposure time was $100 \mathrm{~ms}$. Five control pictures were taken preceding stimulus presentation followed by 20 images during stimulus motion. Another 15 images were taken after cessation of pattern motion.

\section{Image processing}

From the raw fluorescence images, the relative fluorescence changes $(D F / F)$ were determined in the following way. To eliminate the contribution of the autofluorescence of the tissue, the background fluorescence was subtracted from the images. Back- 
ground fluorescence was determined in a rectangular area away from the dyed neuron. To determine DF/F from these backgroundcorrected fluorescence images, the first picture of the sequence was used as a reference and subtracted from each other picture of the sequence. The resulting difference pictures were then divided by the reference picture pixel by pixel. Calculation of $D F / F$ in this way requires a clear distinction of the cellular processes from the background. Otherwise, the denominator of the ratio may become very small and thus the corresponding $\mathrm{DF} / \mathrm{F}$ values will be extremely prone to noise. We therefore determined $\mathrm{DF} / \mathrm{F}$ only for the axon and the major branches by applying a threshold operation to the raw fluorescence images. All pixels within the image with a value smaller than threshold were set to a constant arbitrary value. In some cells filled with fura- 2 , the resting calcium concentration was determined by the ratioing approach (Grynkiewicz et al. 1985). This can be done by relating the DF/F in two pictures taken with excitation filters centered at $380 \mathrm{~nm}$ (bandwidth $10 \mathrm{~nm}$ ) and $349 \mathrm{~nm}$ (bandwidth $10 \mathrm{~nm}$ ), respectively. From the resting calcium concentration, the calcium concentration induced by visual motion can be calculated for each image of the sequence taken at $380 \mathrm{~nm}$ (Lev-Ram et al. 1992; Regehr and Tank 1992; Vranesic and Knöpfel 1991).

\section{Visual stimulation}

The fly faced the inside of a translucent hemispherical screen. The moving patterns were generated mechanically by a rotating striped cylinder with the light source (HBO 100-W mercury arc lamp) placed in its center. The patterns were projected onto the hemisphere from the outside and could be turned about the axis of projection by dove prisms. Two pattern windows with diameters of $\sim 20^{\circ}$, as seen by the fly, could be positioned on arbitrary sites on the hemisphere. The pattern windows were approximately circular. However, depending on which parts of the hemispherical screen they were projected onto, their shape was distorted to an ellipsoid. Accordingly, the grating pattern within the windows was also slightly distorted. On average, the spatial wavelength of the pattern amounted to $\sim 10^{\circ}$. The contrast was $\sim 0.8$. However, when the preparation was illuminated from above during optical recording, it could not be avoided that pattern contrast was reduced by stray light. Within the windows, the patterns could be moved in any direction at variable speeds.

\section{RESULTS}

All data presented in the following represent the $\mathrm{DF} / \mathrm{F}$ induced by stimulation of cells filled with either fura- 2 or Calcium Green. The fluorescence changes were determined from background-subtracted images obtained at one excitation wavelength only ( $380 \mathrm{~nm}$ for fura- $2,472 \mathrm{~nm}$ for Calcium Green). Thus they cannot immediately be converted into absolute calcium concentrations. Only for the resting state of the cell, absolute calcium concentrations were determined with the ratioing method in some cells filled with fura-2. By assuming a dissociation constant of $225 \mathrm{nM}$, they were found to be between 20 and $60 \mathrm{nM}$. On this basis the calcium concentration during stimulation of the cell can be calculated (see above). With a resting calcium concentration of $50 \mathrm{nM}$, a $10 \%$ decrease in relative fluorescence in fura-2-filled cells corresponds to a calcium concentration of $84 \mathrm{nM}$ and a $30 \%$ decrease to a calcium concentration of $185 \mathrm{nM}$.

\section{Spatial distribution of intracellular calcium accumulation}

Visual motion in the preferred direction of a tangential cell leads to pronounced intracellular calcium accumulation.
This accumulation is not homogeneously distributed allover the cell but is confined to only certain parts of it. This is illustrated in Fig. 1. Here, the color-coded image of the DF/ $\mathrm{F}$ in one of the HS cells, the HSN cell, is shown after the cell has been stimulated for $7.5 \mathrm{~s}$ by motion from the front to the back, i.e., the cell's preferred direction. Only the main cellular branches are displayed after the raw fluorescence image was subjected to thresholding, leaving out those parts that were not sufficiently distinct from background fluorescence. Because the cell was injected with fura-2, negative fluorescence changes correspond to an increase in calcium concentration. In this example the cell was depolarized by motion within both stimulus windows that were positioned side by side and thus covered large parts of the cell's receptive field. Motion stimulation leads to a pronounced calcium accumulation in the terminal region of the axon, the soma, and the distal branches of the dendritic tree. Virtually no fluorescence changes can be observed in most parts of the axon or in the proximal dendritic branches. A similar spatial pattern of motion-induced DF/F is observed in other tangential cells (Borst and Egelhaaf 1992; Egelhaaf et al. 1993 ). Quantitative differences in the labeling of cells, even of the same type, may occur. Sometimes, motion-induced relative fluorescence can also be detected in the axon, although here, in general, it is weaker than in the axon terminal, the soma, and the distal dendritic branches (see below). Nevertheless, the spatial pattern of calcium accumulation is basically the same in all experiments performed so far. This shows that the tangential cells of the fly are compartmentalized with respect to calcium accumulation induced by visual motion stimulation.

\section{Calcium accumulation during motion in different directions}

The tangential cells depolarize during motion in their preferred direction and hyperpolarize during motion in the opposite direction. Depending on the velocity, contrast, or spatial structure of the pattern, the magnitude of hyperpolarization during null-direction motion may be almost as large as the magnitude of depolarization during preferred-direction motion, leading to an extremely pronounced direction selectivity of the tangential cells (Egelhaaf et al. 1989). Whether the calcium signal behaves in a similiar way to the electrical signal was investigated in the experiment shown in Fig. 2. The cells were injected with fura- 2 and alternately stimulated with motion in their preferred and null direction. Between stimulation with both directions of motion, there was an interval of $\geq 2$ min to allow the cell to resume to its resting state. To increase the signal-to-noise ratio, the data shown in Fig. 2 were obtained by spatially averaging over the main branches of the whole dendritic tree and by temporal averaging over the entire period of motion stimulation. However, qualitatively the same results are obtained by taking only a more restricted part of the dendrite or single frames of the time-dependent series of fluorescent pictures into account. Whereas motion in the preferred direction leads to a large decrease in relative fluorescence, indicating a significant increase in calcium concentration, the dendritic fluorescence virtually does not change at all during motion 


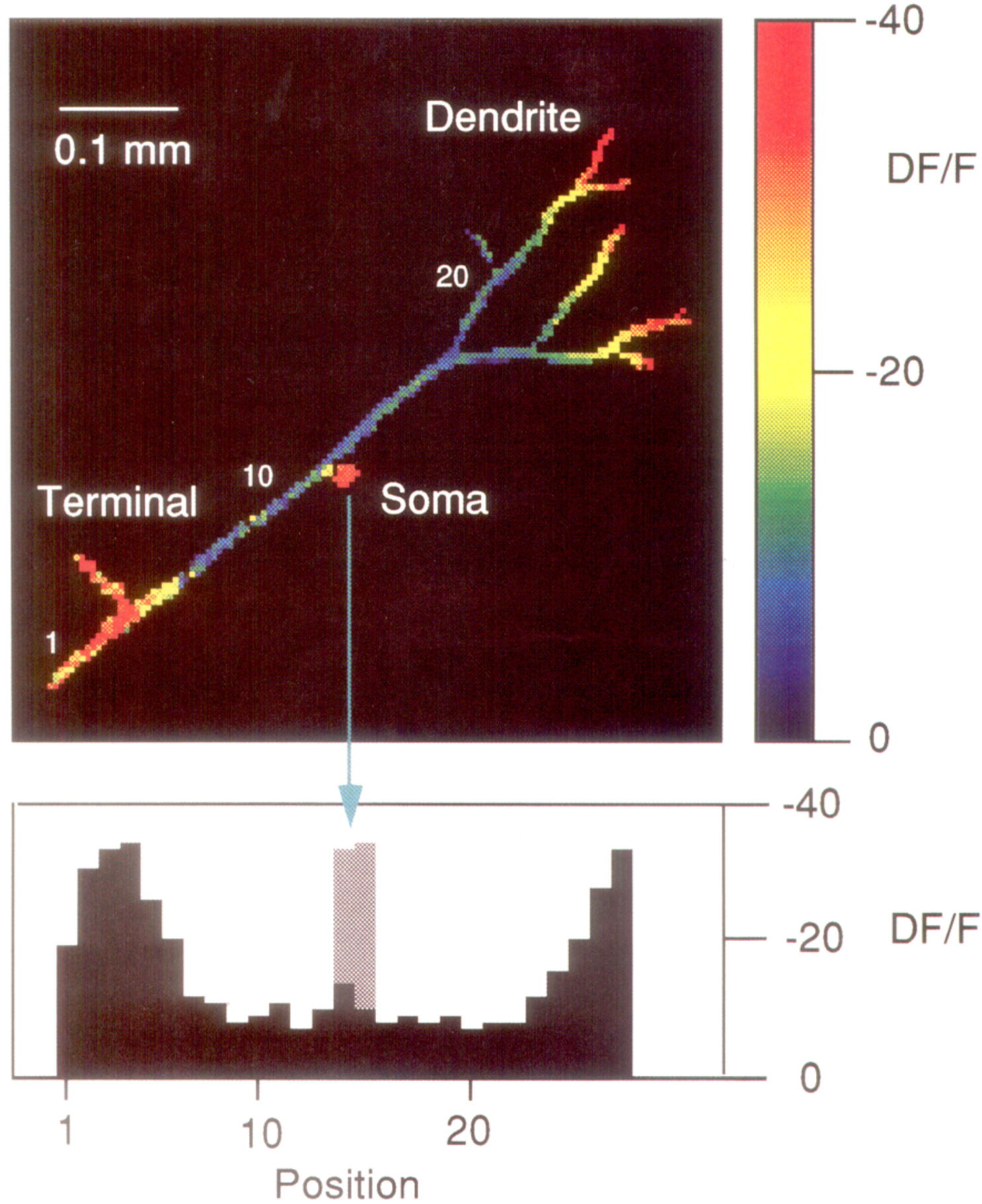

FIG. 1. Spatially resolved sites of calcium accumulation in a tangential cell. Top: axon with its main terminal branches, the main dendritic branches, and the soma, after those parts of the image that did not sufficiently differ from the background were set to 0 . The cell was excited by visual motion in its preferred direction in large parts of its excitatory receptive field. The changes in relative fluorescence (DF/F) (given in \% values) at $7.5 \mathrm{~s}$ after the onset of motion are shown in a colorcoded form, with warmer colors indicating larger negative DF/F. Bottom: DF/F (in \% values) along the entire length of the axon and 1 dendritic branch (black) as well as in the soma (gray). Position values refer to the numbers given in the top diagram. The cell was filled with fura- 2 and the pictures were taken at an excitation wavelength of $380 \mathrm{~nm}$. Thus negative fluorescence changes indicate an increase in calcium concentration. During visual motion stimulation calcium does not accumulate homogeneously all over the cell but preferentially in the axon terminal, the soma, and the distal dendrites.

in the null direction. Although this is true on average, a significant increase in fluorescence and thus a decrease in intracellular calcium concentration could be detected in one example during null-direction motion. Nonetheless, although the motion-induced depolarizations of the cell go along with pronounced changes in the dendritic calcium concentration, the hyperpolarizations, on average, do not much affect the calcium level. 


\section{Preferred Direction Null Direction}

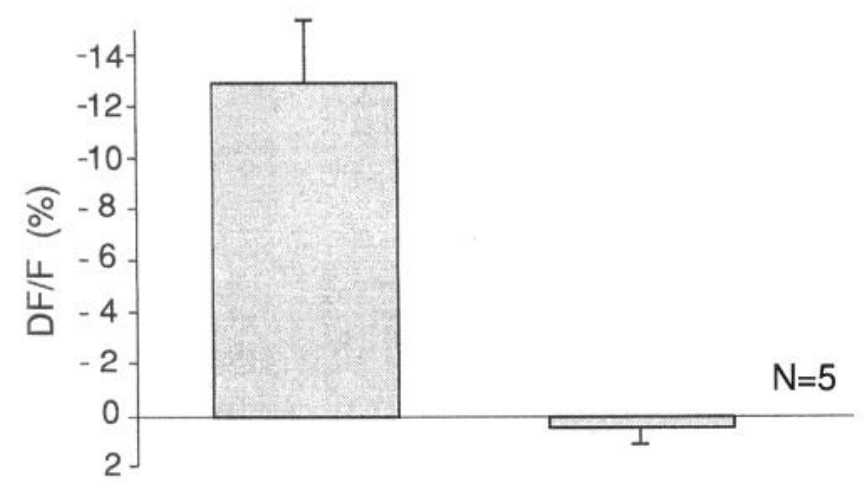

FIG. 2. DF/F induced by preferred-direction motion (left column) and null-direction motion (right column). The data are the mean values and standard deviations obtained from 5 horizontal-motion-sensitive cells with their receptive fields in the medial part of the visual field of the ipsilateral eye (HSE cells) filled with fura-2. Averages were taken over the main branches of the dendritic tree and the entire time interval of motion stimulation. There is a pronounced calcium accumulation in the dendritic tree during motion in the preferred direction. Virtually no change in calcium concentration is observed during null-direction motion.

\section{Localized dendritic calcium accumulation during motion in small parts of the receptive field}

Depending on the size and spatial arrangement of the motion stimuli within the cell's receptive field, it is possible to elicit distinct patterns of calcium accumulation in the dendritic tree. In the experiment shown in Fig. 3 an HSE cell was stimulated by three stimulus configurations, either by motion in the dorsal part of the receptive field, motion in the ventral part, or motion simultaneously in both parts. The cell was injected with Calcium Green and the DF/F was determined in the main dendritic branches. Whereas motion in the upper part of the receptive field leads to an increase in fluorescence and thus an increase in calcium concentration exclusively in the cell's dorsal branches, motion in the lower part of the receptive field leads to an increase in calcium concentration in the ventral dendritic branches. Accordingly, simultaneous motion in both stimulation windows induces a rise in calcium concentration in both ventral and dorsal branches. The calcium concentration does not change much in the middle dendritic branches. This experiment, in accordance with our earlier results (Borst and Egelhaaf 1992; Egelhaaf et al. 1993), shows two important points. 1) Motion in the visual world appears to be projected like a two-dimensional image on the dendritic tree of the tangential cells. This illustrates that the input to the tangential cells is organized in a strictly retinotopic fashion. 2) The calcium accumulation may stay localized even, as in the experiment shown here, after $7.5 \mathrm{~s}$ of motion stimulation. It should be noted, however, that in some experiments the calcium did not stay as localized and after some seconds an increase in calcium concentration may be observed in larger parts of the dendrite. The reasons for this variability are unclear so far.

The overall calcium signal elicited by simultaneous motion in two directly adjacent but nonoverlapping stimulation windows was less than the sum of the responses to motion in the two windows alone. In this respect the calcium signal behaves in a similarly nonlinear way to the corresponding electrical signal of the cell. This is illustrated in Fig. 4, where the calcium signal and the simultaneously determined membrane potential changes are compared at two temporal frequencies of the motion stimulus. The data were obtained by averaging the respective signals over the whole period of motion stimulation and, in the case of the calcium signal, the major branches of the entire dendritic tree. The averaging over the whole dendrite was done because the overall changes in the calcium signal had to be determined. The temporal averaging was done to increase the signal-to-noise ratio. However, in controls it could be assured that qualitatively the same results were obtained when shorter time intervals after the motion onset were taken into account. To allow for a direct comparison of membrane potential and calcium signal, the responses were normalized to the respective largest response amplitudes that were obtained in these experiments. With the pattern moving at a temporal frequency of $2 \mathrm{~Hz}$ in only one stimulation window, the $\mathrm{DF} / \mathrm{F}$ due to stimulus-induced calcium accumulation range between $\sim 70$ and $80 \%$ of the fluorescence changes induced by simultaneous motion in both windows. Thus the calcium signal obtained during combined stimulation in both parts of the receptive field is less than the sum of the calcium signals induced by each elemental stimulus alone. The corresponding changes in membrane potential depend in basically the same way on the spatial extent of the stimulus pattern (compare dotted and black columns in Fig. 4; see also Egelhaaf 1985; Haag et al. 1992; Hausen 1982b). Although the membrane potential changes are only slightly reduced when the stimulus pattern moves with a temporal frequency of $0.1 \mathrm{~Hz}$, the corresponding calcium signals are significantly smaller than at $2 \mathrm{~Hz}$. Nevertheless, also under these conditions, the calcium signals induced by motion in the upper and lower part of the receptive field sum up less than linearly. Finally, it should be mentioned that individual HS cells showed different behavior from the others, i.e., over linear spatial summation of the calcium signal, in a similar way to that found in the centrifugal horizontal $(\mathrm{CH})$ cells, another type of tangential neurons in the fly third visual neuropil (Egelhaaf et al. 1993). However, most HS cells showed under linear spatial summation, as is reflected by the averaged data presented in Fig. 4. The reasons for these differences are currently under investigation.

\section{Time course of calcium accumulation}

The degree of localization of calcium accumulation depends on the time that has passed after the onset of motion stimulation. This is illustrated in Fig. 5, where the time course of $\mathrm{DF} / \mathrm{F}$ is displayed at five locations along one major branch of the dendritic tree. In three respects the pattern of calcium accumulation varies in a graded way along the dendritic branch. 1) The maximal amplitude that is reached continually decreases from the distal to the proximal part of the dendrite. 2) The maximum is reached much earlier in the distal part of the dendrite than in the proximal ones, where it increases until the pattern stops moving. 3) In the distal parts of the dendritic tree, the calcium concentration starts to decline just after the stimulus stops moving, whereas 

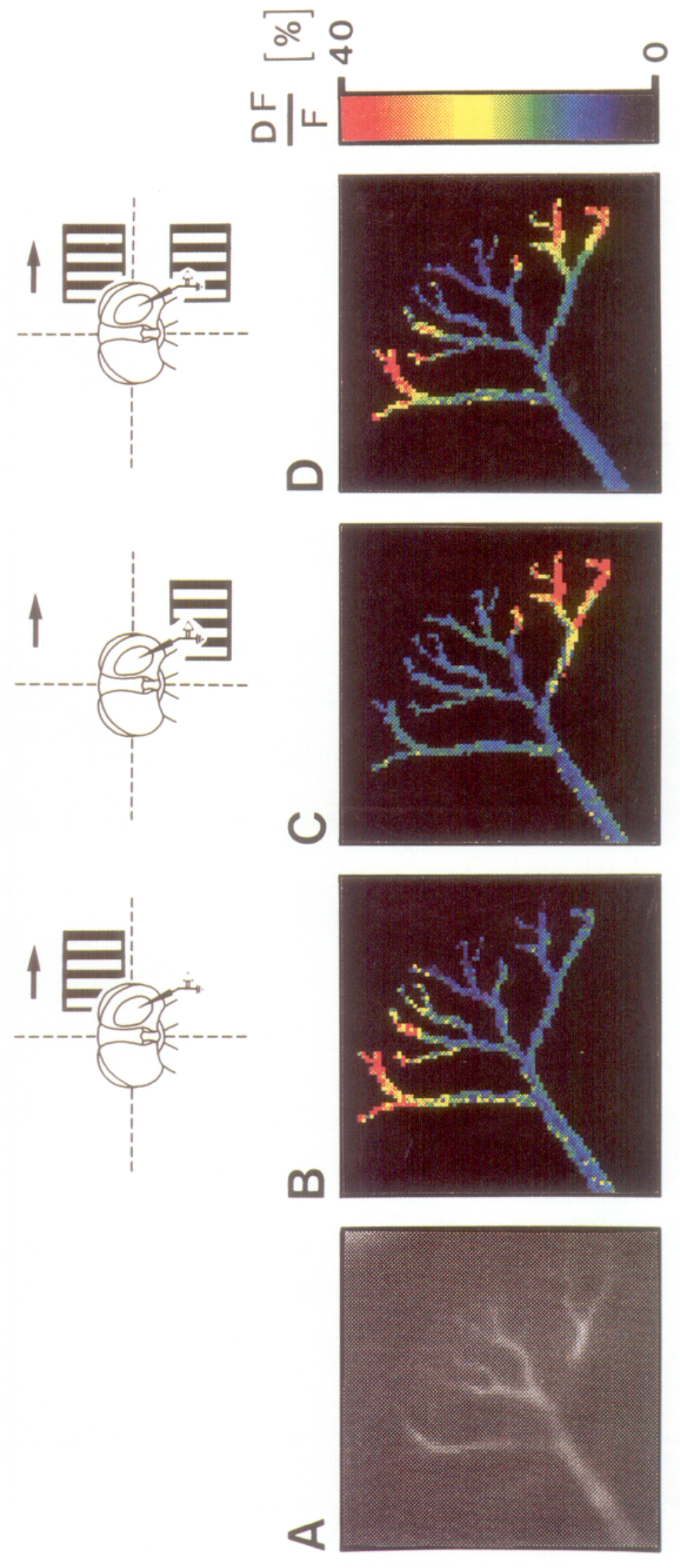

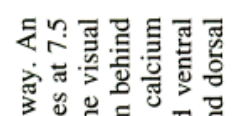

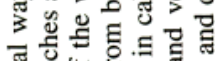

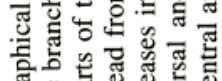

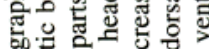

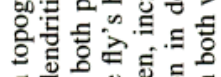

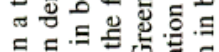

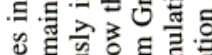

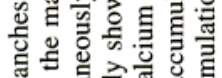

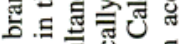

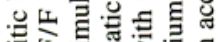

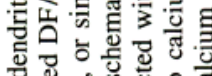

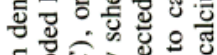
. 흥흐물

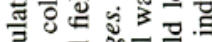

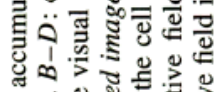

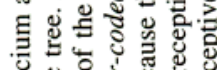
를 둥

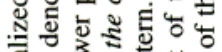

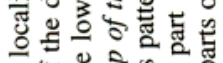
응 올

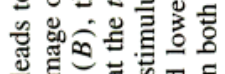

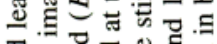

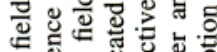

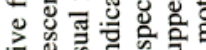

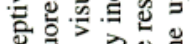

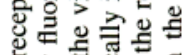
死

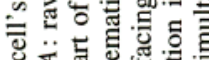

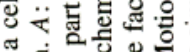
过芯出 는을 믈.

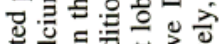

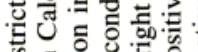

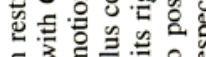

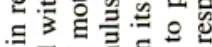

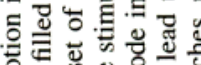

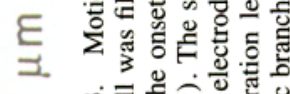

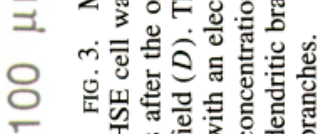



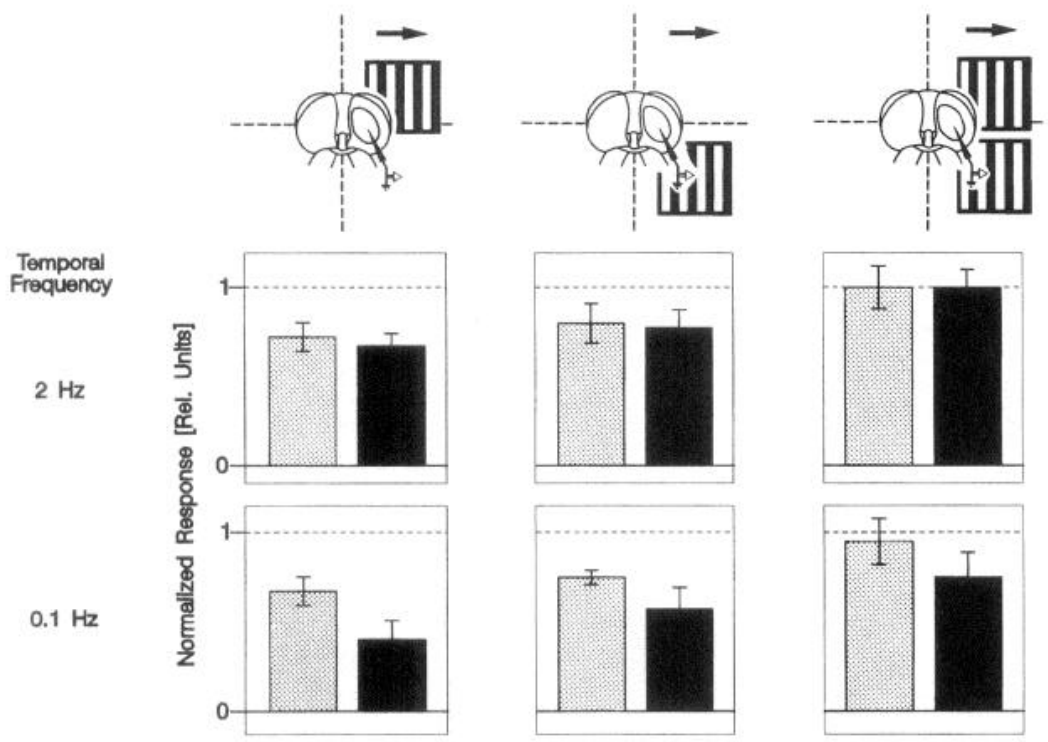

$N=5$
Membrane Potential

Calclum Signal

$2 \mathrm{mV}$
$T-4 \%$

FIG. 4. Comparison of calcium signals (black columns) and membrane potential changes (dotted columns) of horizontal-motion-sensitive (HS) cells during motion in the upper and lower part of the cell's receptive field or simultaneous motion in both parts (schematically indicated by insets at top of the data). The responses were induced by motion stimulation in the cell's preferred direction either at a temporal frequency of either 2 or $0.1 \mathrm{~Hz}$. The data are means \pm SE obtained from HSE cells in 5 flies. The cells were filled with fura- 2 . The data were obtained by averaging the respective signals over the whole period of motion stimulations and, in the case of the calcium signal, the major branches of the dendritic tree. To allow direct comparison of both signals, the responses were normalized with respect to the largest response amplitudes that were obtained in these experiments. Note that in contrast to the experiment shown in Fig. 3, the two stimulation areas are directly adjacent to each other. On average, the calcium signal elicited by simultaneous motion in both windows is less than the sum of the responses to motion in the 2 windows alone. In this respect the calcium signal behaves in a similarly nonlinear way to the corresponding electrical signal. This is true for both temporal frequencies. However, although the membrane potential changes are only slightly reduced when the stimulus pattern moves at a temporal frequency of $0.1 \mathrm{~Hz}$, the corresponding calcium signals are significantly smaller than at $2 \mathrm{~Hz}$. in the proximal ones it stays at a constant plateau level at least until the end of the time interval of the experiment.

In contrast to the electrical signal, which reaches its maximum value within $\sim 10 \mathrm{~ms}$, calcium accumulates only relatively slowly during the course of motion stimulation. This difference is illustrated in Fig. 6, where the membrane potential change induced in an HSE cell filled with fura-2 is compared with the simultaneously determined fluorescence changes. The electrical signal was recorded with a microelectrode in the axon of the cell right between the main bifurcation point of the dendrite and the axon terminal. The fluorescence changes were monitored with a photodiode

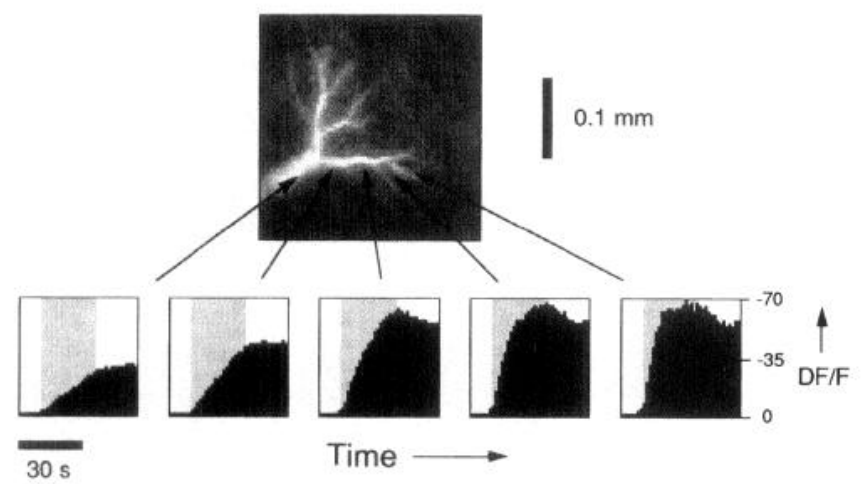

FIG. 5. Time course of calcium accumulation in different locations along a dendritic branch. Top: raw fluorescence image of an HSE cell filled with fura-2. Bottom panels: time course of the $\mathrm{DF} / \mathrm{F}$ at the dendritic locations indicated by the arrows. The DF/F are the average over a quadrate array of 9 pixels. During the time interval indicated by the shaded area, the cell was activated by a stimulus pattern moving in its preferred direction. Negative DF/F correspond to increases in calcium concentration. During motion stimulation, calcium accumulates slowly along the entire dendritic branch. However, it accumulates with a smaller time constant and reaches a much larger level in the distal dendritic branches than in the proximal ones. placed over the dendritic tree of the cell rather than with the CCD camera because of the much better temporal resolution. It should be noted that despite the different time courses, the calcium signal starts at the same time as the motioninduced membrane potential changes.

\section{Dependence of calcium and electrical signal on the temporal frequency of pattern motion}

The electrical response of the tangential cells does not depend exclusively on the velocity of the pattern but in

\section{Calcium Signal}
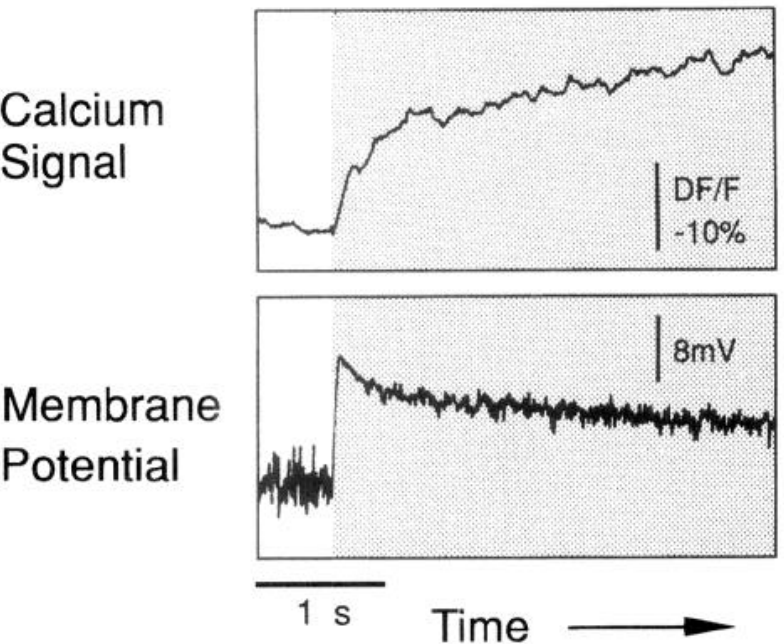

FIG. 6. Comparison of calcium accumulation and the simultaneously recorded membrane potential changes induced at the onset of motion stimulation. The cell was filled with fura- 2 . The time course of the calcium signal was determined with a photodiode. Shaded area: time of motion stimulation. Negative fluorescence changes indicate increases in calcium concentration. Although the calcium concentration and the membrane potential start at the same time, they change with a different velocity. 
Calcium Signal

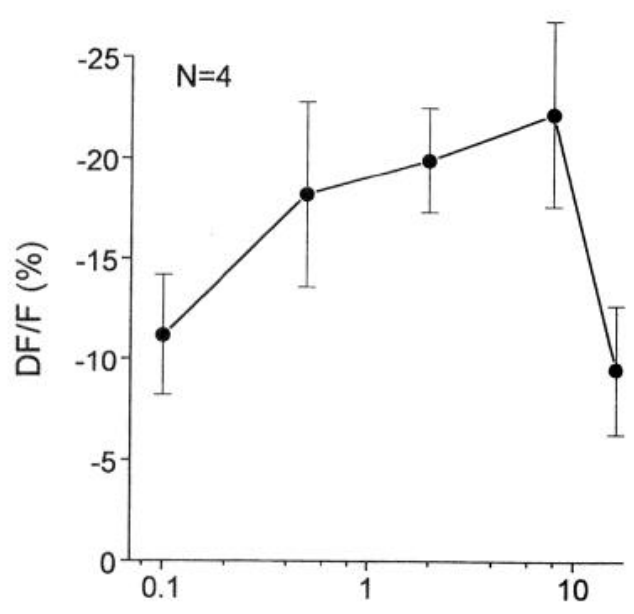

Membrane Potential

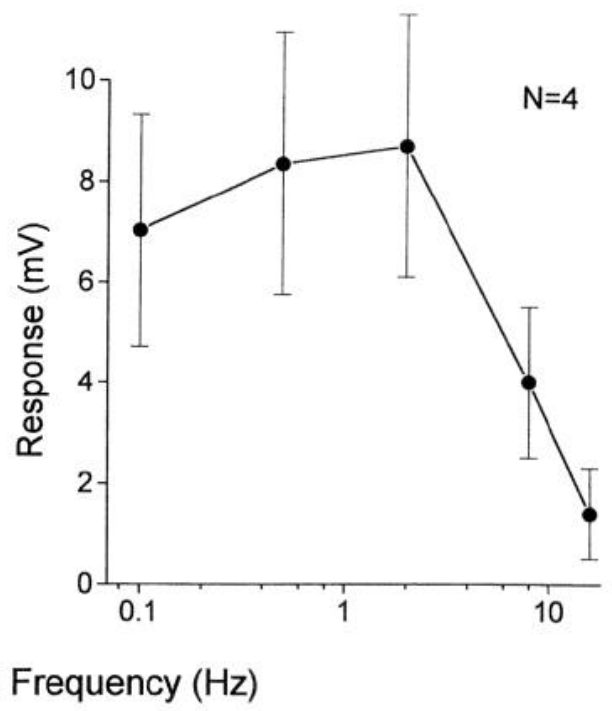

FIG. 7. Dependence of the mean calcium signal and the mean membrane potential on the temporal frequency of stimulus motion. The membrane potential changes of 4 HSE cells filled with fura- 2 were determined simultaneously with the DF/F. The responses were averaged over the whole period of motion stimulation, the optical signals in addition over all major branches of the dendritic tree. Mean values and standard deviations are given. Negative DF/F correpond to increases in intracellular calcium concentration. Both the intracellular calcium concentration and the membrane potential changes increase with increasing temporal frequency, reach a maximum, and then decrease again. The locations of the maxima of both responses differ.

addition on various pattern parameters such as contrast and spatial frequency (Egelhaaf and Borst 1989, 1990; Egelhaaf et al. 1989; Hausen 1982b; Lenting et al. 1984; Maddess 1986; Maddess and Laughlin 1985). Most importantly, for grating patterns with sinusoidal intensitiy modulation, the velocity at which maximum responses are obtained is indirectly proportional to the spatial frequency of the pattern leading to a constant response optimum at a certain temporal stimulus frequency (Eckert 1980; Egelhaaf and Borst 1993a; Götz 1972). So the question arises of whether the motioninduced calcium accumulation depends in a similar way on these stimulus parameters as does the membrane potential measured in the axon.

In Fig. 7 we compare how the dendritic calcium signal and the simultaneously recorded axonal membrane potential changes depend on the temporal frequency of the stimulus pattern. The data are mean values and standard deviations of the responses averaged over the entire time interval of motion stimulation. They were obtained from four HSE cells filled with fura-2. For both the calcium and the electrical signal, the response amplitude initially increases with increasing temporal frequency, reaches a maximum, and then declines again. However, the response optimum is located at different temporal frequencies for both types of responses. Whereas the electrical signal peaks around a frequency of $1 \mathrm{~Hz}$, the calcium signal shows a broad optimum between 1 and $10 \mathrm{~Hz}$. Thus, although the calcium signal depends on the temporal frequency in a similar way as does the membrane potential, distinct differences between these two indicators of neural activity are obvious. At the lower temporal frequencies, the membrane potential changes are relatively larger than the calcium signals (see also Fig. 4); the reverse is true in the high temporal frequency range that was tested.
Where do these differences come from? As was already shown in Figs. 5 and 6, the time course of the calcium signal at the onset of motion stimulation differs considerably from the corresponding changes in membrane potential. However, pronounced differences may also be found during maintained motion, depending on the dynamic properties of the stimulus. This is illustrated in Fig. 8 for one of the HSE cells that were used to determine the temporal frequency dependence of the time-averaged responses shown in Fig. 7. Qualitatively the same results were obtained for the other three HSE cells. The calcium signal and the membrane po-

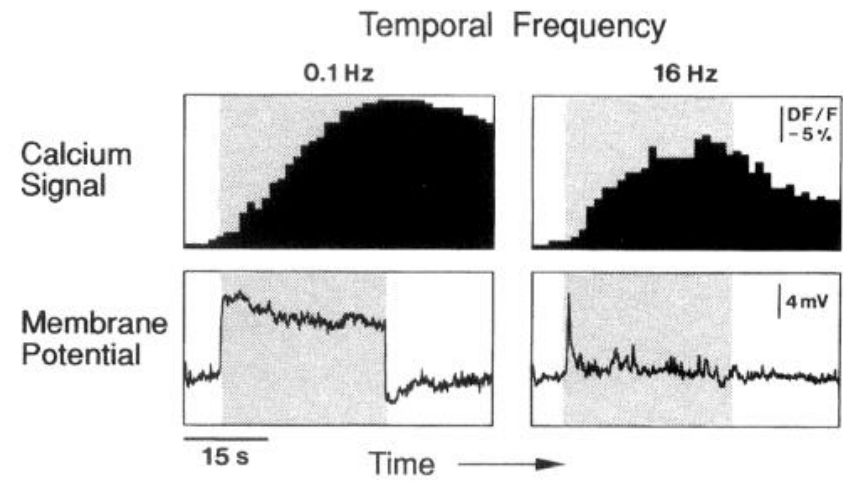

FIG. 8. Time course of the calcium signal and the electrical response at a low and a high temporal frequency. An HSE cell was injected with fura2. Shaded area: time interval of motion stimulation. The optical signals are averages taken over the main branches of the dendritic tree. Negative fluorescence changes correspond to an increasing calcium concentration. There is only a transient membrane potential change at $16 \mathrm{~Hz}$, whereas at $0.1 \mathrm{~Hz}$ the cell stays depolarized during maintained motion. In contrast, at both temporal frequencies the calcium signal starts to decrease only after the motion stimulus terminates. 
tential changes induced by motion with two different temporal frequencies are displayed versus time. The optical signals are averages taken over the main dendritic branches. At 0.1 $\mathrm{Hz}$, the membrane potential, after a transient period, settles at a steady-state level of maintained depolarization. In contrast, at $16 \mathrm{~Hz}$, the electrical signal only exhibits a brief response transient, whereas the steady-state response level is almost indistinguishable from the resting membrane potential. The finding that the transient and steady-state responses of directionally selective motion-sensitive neurons depend in a different way on temporal frequency is in accordance with predictions by the motion detector theory (Borst and Bahde 1986; Egelhaaf and Borst 1989, 1990) and also with previous electrophysiological data (Egelhaaf and Borst 1989, 1990; Hausen 1982b; Maddess and Laughlin 1985). As was already shown in Fig. 6, the calcium signal at $0.1 \mathrm{~Hz}$ resembles a low-pass-filtered version of the corresponding membrane potential changes in that it creeps up over many seconds and only starts to decline when motion stimulation has stopped. At $16 \mathrm{~Hz}$ stimulus frequency, the time course of the calcium signal does not differ much from the time course obtained at $0.1 \mathrm{~Hz}$, although the corresponding electrical signals differ considerably. It again increases as long as the motion stimulus persists and only declines after the stimulus stops moving. It is obvious that this difference in time course results in different temporal frequency optima for the electrical and calcum signal.

\section{Voltage-dependent calcium channels}

This finding raises the question of in which way the calcium signal is related to the electrical signal. Because for methodological convenience all electrical measurements were made in the axon, while, except in Fig. 1, we analyzed the visually induced calcium accumulation in the dendritic tree, quantitative differences between both indicators of neural excitability are not too surprising. In principle, the observed changes in free cytosolic calcium concentration can be caused by three different mechansims that are not mutually exclusive. 1) Calcium could enter the cell from the outside through voltage-dependent channels. 2) In the dendrite, calcium could enter the cell from the outside through transmitter-gated channels. 3) Calcium could be relased from internal stores. One way to decide between these three possibilities is to change the membrane potential not by visual motion but by current injection. However, because the cell cannot be assumed to be isopotential (Haag et al. 1992), current injection in the axon might only allow for a poor control over the membrane potential in the dendrite. Therefore an electrode was inserted into one of the dendritic branches (a VS1 cell in Fig. 9, top diagram). The cell was filled with fura- 2 and then activated alternately by motion stimulation or by injecting depolarizing current via the electrode (in the experiment shown here, $\sim 3 \mathrm{nA}$ ). The time course of the calcium signal was determined at three dendritic sites. It is obvious that calcium does not only accumulate in the dendrite during visual motion stimulation, but also, in a similar way, during depolarization of the cell with current. The current-induced calcium accumulation demonstrates the existence of voltage-gated calcium channels through which calcium rushes into the cell when activated by the membrane potential.

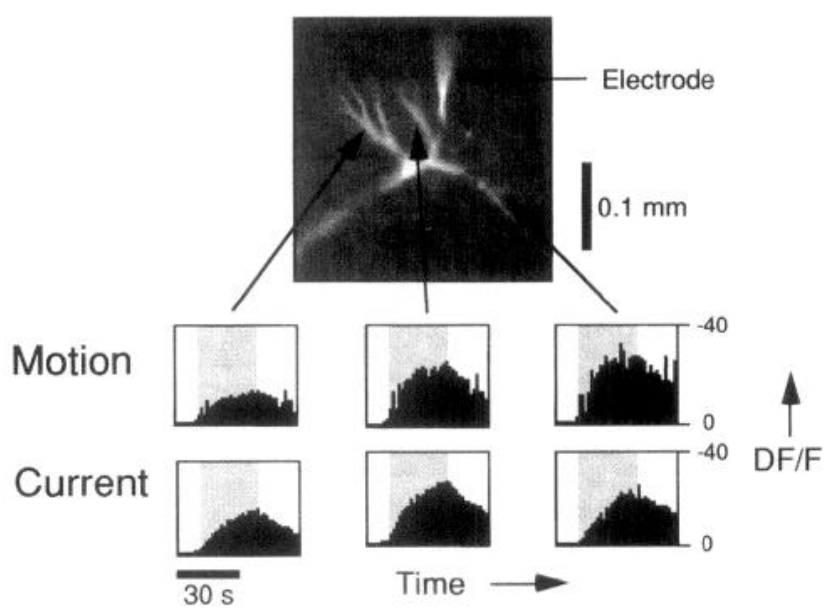

FIG. 9. Dendritic calcium accumlation during motion stimulation and direct depolarization of the cell by current injection. Top: raw fluorescence image of a vertical-motion-sensitive, frontally located (VS1) cell that was filled with fura- 2 . The microelectrode can be seen inserted into 1 of the major dendritic branches. The time course of the calcium signal was determined at 3 dendritic sites as marked by arrows $(3 \times 3$ pixels each $)$. The cell was stimulated either by visual motion or by depolarizing current $(\sim 3$ $\mathrm{nA}$ ) that was injected into the dendrite via the microelectrode. Shaded areas in data plots: time interval in which the cell was activated. Increases in calcium concentration correspond to negative DF/F. Calcium does not only accumulate in the dendrite during visual motion stimulation but also during depolarization of the cell with current, suggesting the existence of voltagedependent calcium channels.

\section{DISCUSSION}

By applying optical recording techniques to a class of directionally selective motion-sensitive neurons in the visual system of the fly that are involved in controlling optomotor responses, we could show under in vivo conditions that pronounced calcium accumulations are elicited in the dendritic tree of these cells by visual motion stimulation (Borst and Egelhaaf 1992). These initial experiments as well as the more detailed investigations of the present study led to the following conclusions. 1) The tangential cells are compartmentalized with respect to the accumulation of calcium during visual motion stimulation, in that calcium preferentially accumulates in the axon terminal, the soma, and the dendritic tree (Fig. 1). During null-direction motion, no change in calcium concentration is observed (Fig. 2). 2) Dendritic calcium accumulation intially is confined to those parts of the dendrite that are close to the site of direct synaptic activation. However, depending on the stimulus strength, calcium may subsequently accumulate also in other parts of the dendrite and even in the axon (Figs. 3 and 5). 3) Like the electrical signal, the calcium signal elicited in different branches of the dendritic tree summates nonlinearly according to a saturation nonlinearity; this is true at least for large response amplitudes (Fig. 4). Although calcium accumulation starts right with the onset of motion, its time course is much slower than the corresponding membrane potential changes, which, depending on the temporal frequency of the stimulus, may even be completely transient (Figs. 6 and 8). 4) The strength of calcium accumulation depends on the parameters of the visual stimulus, such as temporal frequency, in a similar way to the electrical signal of the cell. However, at high stimulus velocities characteristic differences are obvious (Fig. 7). 5) 
Depolarization of the cell with current injected through a microelectrode leads to calcium accumulation in the dendrite, suggesting that there are voltage-dependent calcium channels (Fig. 9). Therefore it is plausible to assume that also during motion stimulation, calcium accumulates in the cell via voltage-gated calcium channels.

These findings raise two questions. 1) Which mechanisms lead to calcium accumulation in the different parts of the tangential cells during motion stimulation? 2) What is the function of calcium in these cells? These two issues are discussed in the following.

\section{How does calcium accumulate in the tangential cells?}

Because motion stimulation and depolarization of the dendrite by current injected via the electrode lead to similar increases in intracellular calcium concentration, it can be assumed that calcium enters the cell through voltage-dependent calcium channels. Of course, this does not exclude that, in addition, calcium is also released from intracellular stores mediated during visual stimulation by second messengers or, in the dendrite, enters the cell also through transmittergated channels. Although nothing is known so far, apart from photoreceptors (Hardie 1991; Hardie and Minke 1992; Sandler and Kirschfeld 1991), about the properties of calcium channels in the fly, a large number of voltage-gated calcium channels has been characterized in other systems (e.g., Swandulla et al. 1991; Tsien et al. 1988).

There are various possible reasons why during visual large-field motion calcium accumulates mainly in the dendrite, the soma, and the axon terminal. I) This could be due to an inhomogeneous calcium channel densitiy in the different parts of the neuron. 2) The inhomogeneous calcium distribution could be the consequence of gradients in the surface-to-volume ratio along the cellular processes. 3 ) The spatial pattern of calcium concentration may be affected by the properties of the intrinsic calcium buffers and pumps. Therefore, to quantitatively assess differences in visually induced calcium concentration in the tangential cells in terms of channel densitiy variations requires correction of the experimental data for all these factors, which is not possible at present.

Nevertheless, the present data allow us to discard the possibility that differences in the surface-to-volume ratio are the main determinant of the spatially inhomogeneous calcium pattern during motion stimulation. This is because it would then be expected that the visually induced changes in calcium concentration are the more pronounced the thinner the neuronal processes are. However, this is obviously not the case, because there are also pronounced changes in calcium concentration in the terminal part of the axon, the soma, and the main dendritic branches, which have about the same diamter or even a larger diameter than those parts of the axon where not much calcium accumulation can be observed.

From the retinotopic calcium accumulation found by stimulation of localized parts of the cell's receptive field, it can be concluded that calcium enters the dendrite initially in those branches that are close to the postsynaptic sites of the corresponding local input elements. One possible way to explain the slower and delayed increase in calcium concen- tration and the smaller calcium levels at increasingly distant sites of the dendrite is to assume that calcium spreads out within the cell. Model simulations based on the geometric properties of the tangential cells and using published diffusion coefficients for calcium (Baylor and Hollingworth 1988 ) could show that this may well be possible (unpublished data). It should be noted, however, that this is not meant to imply that calcium spreads within the tangential cells by simple diffusion, because nerve cells are highly compartmentalized structures and contain a wealth of complex transport and calcium buffer systems (e.g., Kasai and Petersen 1994). The time course of calcium accumulation at different sites in the fly tangential cells is rather similar to what has been observed in barnacle photoreceptors, where calcium enters the cell in the tips of the axon terminal, from which it spreads along the axon (Callaway et al. 1993). This makes it suggestive to speculate that intracellular spread of calcium may also play a role in shaping the dynamics of calcium accumulation in those parts of the dendrite that are not directly activated by synaptic input.

Irrespective of the mechanism by which calcium accumulates in the tangential cells, the calcium gradients are much steeper than is likely to be the case for the gradients in membrane potential. On the basis of electrophysiological measurements of the motion-induced membrane potential at various locations of the axon and the dendritic tree of VS cells, it is suggested that these cells have a length constant in the range of $\sim 0.5 \mathrm{~mm}$ ( $\mathrm{J}$. Haag, unpublished data). In any case, in all tangential cells with graded membrane potential changes as their main response mode, pronounced potential changes can be recorded during visual motion all along the axon, although virtually no changes in calcium concentration can be observed there. This suggests a nonlinear transformation of the membrane potential into local changes of calcium concentration. One source of this nonlinear transformation may be an inhomogeneous distribution of calcium channels. However, because in the dendrite calcium accumulation may stay rather localized although calcium channels are present in all dendritic branches, a nonlinear activation curve of the calcium channels may be assumed. In other systems, the activation of calcium channels often rises steeply when a given membrane potential level is reached. As a consequence, the spatial pattern of membrane potential distribution may differ considerably from the spatial calcium pattern. Thus two different spatial patterns of neuronal activation may coexist in the fly tangential cells during visual motion stimulation.

Although this reasoning may provide a plausible explanation for the spatially inhomogeneous calcium distribution in the tangential cells, the time course of the calcium signal does not readily follow from it. There are two major discrepancies between the time course of calcium accumulation and of the membrane potential changes. 1) Although the onset of calcium accumulation starts at the same time as the depolarization of the cell, it rises much more slowly and may reach its steady-state level several seconds later than the membrane potential. 2) At high temporal frequencies of pattern motion, the membrane potential deviates from its resting level only during the onset of motion, whereas the calcium signal stays at a steady-state level well above the resting level throughout the stimulus period. 
The time-dependent changes in intracellular calcium are determined on the one hand by the time course of entry into the cells, and on the other hand by the kinetics of the removal or buffering processes. Although the removal of calcium was concluded to be slow compared with the calcium entry (Ross and Werman 1987; Ross et al. 1986), it cannot be taken for granted that the slow time course of DF/F faithfully reflects equally slow changes in intracellular calcium concentration. With its low dissociation constant, fura-2 itself is a calcium buffer that may well compete at high concentrations with intrinsic calcium binding proteins and thus may affect the calcium dynamics. This has been shown in various studies by comparing the calcium signals obtained with different calcium sensors or different amounts of injected fura2 (Baylor and Hollingworth 1988; Miyakawa et al. 1992; Regehr and Tank 1992). To obtain good signal-to-noise calcium measurements in the presence of high brackground fluorescence levels, it was necessary to inject enough fura2 into the cell to make at least the main dendritic branches visible. Without systematic analysis it is hardly possible to assess whether at these fura- 2 concentrations the calcium dynamics was already affected significantly by fura- 2 buffering. Nonetheless, we did not observe any systematic differences in the time course of the calcium signal between cells that were filled with fura- 2 quite well and cells that were only poorly filled as judged by the visibility of their dendritic branches in the raw fluorescence images.

However, the buffer capabilities of intracellular calcium binding proteins or fura- 2 cannot explain that the calcium level is maintained even if there are only transicnt changes in membrane potential, as during motion stimulation with high temporal frequencies. If the calcium influx is through voltage-dependent calcium channels, how can calcium continue to rush into the cell when the membrane potential is already close to its resting level? The answer to this question may lie in the fact that the axonal membrane potential represents the spatially integrated signals originating from different dendritic branches that receive their input from many local motion-sensitive elements. The output of these local elements is modulated in time depending on the spatial phase of the stimulus pattern that passes their receptive field (Egelhaaf et al. 1989). Because the local motion-sensitive elements that impinge on neighboring branches of the dendritic tree "look" at different spatial phases of the moving pattern, their output modulations are phase-shifted with respect to each other. As a consequence, these modulations disappear when the outputs of a sufficient number of such local elements are spatially pooled (Egelhaaf et al. 1989). In the case of motion at high temporal frequencies, this phase shift may lead to an almost complete cancellation of the temporal modulations in the spatial average and to only a small steadystate response level in the axon. Nonetheless, at any time there are dendritic branches where the local membrane potentials may still be significantly larger than the resting level, whereas in others they may be below the resting level. Because the transformation of membrane potential into calcium accumulation appears to follow a rectification nonlinearity (Fig. 2), this spatiotemporal pattern of membrane potential is likely to result in a net calcium influx into the dendrite. Thus the experimentally observed calcium influx during motion stimuli at high temporal frequencies is not in contradic- tion with the assumption that calcium enters the cell via voltage-dependent channels.

\section{Possible functions of calcium}

In principle, calcium can serve two functions. By crossing the external membrane it leads to local depolarizations of the cell and thus acts as a charge carrier. From there it will diffuse or may be transported by active mechanisms to other parts of the cell where it may act as a second messenger in mediating other physiological responses. Accordingly, the functional significance of motion-induced calcium accumulation in the tangential neurons of the fly may be quite different for the different parts of the cell. In the axon terminal, calcium is likely to serve its usual function as second messenger in mediating transmitter release. Calcium accumulation in the soma came as a surprise, because the soma of insect neurons, which lies outside the mainstream of cellular information processing, is usually regarded as electrically inexcitable. Thus it appears suggestive to speculate that, also in this part of the cell, calcium may play a role as second messenger, perhaps in gene regulation. The functional role of calcium in the dendritic tree may be twofold. I) It may again act as a second messenger inducing long-term changes in excitability or, in cases where the dendrite also houses presynaptic transmitter release sites, it may activate transmitter release. Although at present there are no experimental data to support the first hypothesis, presynaptic sites have recently been revealed in an ultrastructural study in the dendritic tree of one type of tangential cell, the so-called ventral centrifugal horizontal (VCH) cell (Gauck et al. 1994), where calcium has been shown to accumulate in a similar way as in the cells studied in the present paper (Egelhaaf et al. 1993). 2) As a charge carricr, calcium depolarizes the membrane, and thus, depending on its relative contribution to the depolarization of the tangential cells during motion stimulation, may play an important role in dendritic integration of synaptic information. There are recent findings that support this function of calcium for dendritic integration. For patterns with high contrast, the response amplitude increases with an increasing number of activated input channels according to a saturation nonlinearity (Egelhaaf 1985; Haag et al. 1992; Hausen 1982b). Interestingly, a similar saturation nonlinearity was found for the calcium signal at high pattern contrasts. However, when the pattern contrast is low, the measured electrical response may be considerably larger than the one predicted by linear summation of the responses elicited by stimulating the individual input channels alone (Egelhaaf et al. 1994). This may well be the consequence of a mutual amplification of the postsynaptic signals. All these data suggest that although the spatial integration properties of the fly tangential neurons can be explained for large response amplitudes on the basis of cable theory for passive dendritic trees, this is hardly possible for the cells' output at medium response levels, and active facilitatory processes have to be assumed. It is suggestive to speculate that calcium might be involved in such mechanisms. Current experiments are aimed at characterizing the different voltage-dependent currents observed in these neurons electrophysiologically. In combination with further optical recording experiments, these analyses will clarify the role of calcium in dendritic integration in the tangential cells of the fly. 
We are grateful to K. Götz, J. Haag, and A.-K. Warzecha for numerous helpful discussions and for critically reading the manuscript.

Present address and address for reprint requests: M. Egelhaaf, Centre for Visual Sciences, Research School of Biological Sciences, Australian National University, GPO Box 475, Canberra, Act 2601, Australia; present address: A. Borst, Friedrich-Miescher Laboratorium der MPG, Spemannstr. 37-39, D-72076 Tübingen, Germany.

Received 8 July 1994; accepted in final form 27 January 1995.

\section{REFERENCES}

Bausenwein, B., Dittrich, A. P. M., and Fischbach, K.-F. The optic lobe of Drosophila melanogaster. Cell Tissue Res. 267: 17-28, 1992.

BAYloR, S. M. AND Hollingworth, S. Fura-2 calcium transients in frog skeletal muscle fibres. J. Physiol. Lond. 403: 151-192, 1988.

BORST, A. AND BAHDE, S. What kind of movement detector is triggering the landing response of the housefly? Biol. Cybern. 55: 59-69, 1986.

BORST, A. AND EGELHAAF, M. Principles of visual motion detection. Trends Neurosci. 12: 297-306, 1989.

Borst, A. AND EgELHAAF, M. Direction selectivity of fly motion-sensitive neurons is computed in a two-stage process. Proc. Natl. Acad. Sci. USA 87: 9363-9367, 1990.

Borst, A. AND EgELHAAF, M. In vivo imaging of calcium accumulation in fly interneurons as elicited by visual motion stimulation. Proc. Natl. Acad. Sci. USA 89: 4139-4143, 1992.

BORST, A. AND EgelHAAF, M. Detecting visual motion: theory and models. In: Visual Motion and its Role in the Stabilization of Gaze, edited by F. A. Miles and J. Wallman. Amsterdam: Elsevier, 1993.

Callaway, J. C., Lasser-Ross, N., Stuart, A. E., and Ross, W. N. Dynamics of intracellular free calcium concentration in the presynaptic arbors of individual barnacle photoreceptors. J. Neurosci. 13: 11571166, 1993.

Chan, C. Y., Hounsgaard, J., And MidtgaARd, J. Excitatory synaptic responses in turtle cerebellar Purkinje cells. J. Physiol. Lond. 409: 143$156,1989$.

ECKERT, H. Functional properties of the H1-neurone in the third optic ganglion of the blowfly, Phaenicia. J. Comp. Physiol. 135: 29-39, 1980.

EGELHAAF, M. On the neuronal basis of figure-ground discrimination by relative motion in the visual system of the fly. I. Behavioural constraints imposed on the neuronal network and the role of the optomotor system. Biol. Cybern. 52: 123-140, 1985.

EgelhaAF, M. AND Borst, A. Transient and steady-state response properties of movement detectors. J. Opt. Soc. Am. 6: 116-127, 1989.

EgelHAAF, M. AND BoRST, A. Transient and steady-state response properties of movement detectors: errata. J. Opt. Soc. Am. 7: 172, 1990.

EgelHAAF, M. AND BoRST, A. Movement detection in arthropods. In: Motion and its Role in the Stabilization of Gaze, edited by J. Wallman and F. A. Miles. Amsterdam: Elsevier, 1993a.

EGELHAAF, M. AND BORST, A. A look into the cockpit of the fly: visual orientation, algorithms, and identified neurons. J. Neurosci. 13: 4563 4574, 1993 b.

EgelhaAF, M., Borst, A., AND ReICHARDT, W. Computational structure of a biological motion detection system as revealed by local detector analysis in the fly's nervous system. J. Opt. Soc. Am. 6: 1070-1087, 1989.

EgelhaAF, M., Borst, A., Warzecha, A.-K., Fleccks, S., AND WILDEMANN, A. Neural circuit tuning fly visual neurons to motion of small objects. II. Input organization of inhibitory circuit elements by electrophysiological and optical recording techniques. J. Neurophysiol. 69: 340-351, 1993.

EgelhaAF, M., Hausen, K., Reichardt, W., and Wehrhahn, C. Visual course control in flies relies on neuronal computation of object and background motion. Trends Neurosci. 11: 351-358, 1988.

EgelhaAf, M., Warzecha, A.-K., AND Borst, A. Nonlinear interactions between postsynaptic signals in the dendritic tree of motion sensitive neurons in the blowfly Calliphora. In: Sensory Transduction, edited by N. Elsner and H. Breer. Stuttgart, Germany: Thieme, 1994, p. 444.

GAUCK, V., EGELHAAF, M., AND BORST, A. Electronmicroscopical and electrophysiological analysis of synaptic interactions in the circuit mediating figure-ground-discrimination in the blowfly Calliphora. In: Sensory Transduction, edited by N. Elsner and H. Breer. Stuttgart, Germany: Thieme, 1994, p. 445.

Gilbert, C., Penisten, D. K., AND Devoe, R. D. Discrimination of visual motion from flicker by identified neurons in the medulla of the fleshfly
Sarcophaga bullata. J. Comp. Physiol. A Sens. Neural Behav. Physiol. 168: 653-673, 1991.

GöTZ, K. G. Principles of optomotor reactions in insects. Bibl. Ophthalmol. 82: 251-259, 1972.

GRYNkiewicZ, G., PoEnie, M., AND Tsien, R. Y. A new generation of $\mathrm{Ca}^{2+}$ indicators with greatly improved fluorescence properties. J. Biol. Chem. 260: 3440-3450, 1985.

HaAG, J., EgelhaAf, M., AND Borst, A. Dendritic integration of visual motion information in the fly. Neurosci. Lett. 140: 173-176, 1992.

HAAG, J., EGELHAAF, M., AND BORST, A. Active membrane properties of motion-sensitive neurons of the fly Calliphora erythrocephala. In: Gene-Brain-Behaviour, edited by N. Elsner and M. Heisenberg. Stuttgart, Germany: Thieme, 1993, p. 96.

HARDIE, R. AND MINKE, B. The trp gene is essential for a light-activated $\mathrm{Ca} 2+$ channel in Drosophila photoreceptors. Neuron 8: 643-651, 1992.

HARDIE, R. C. Whole-cell recordings of the light induced current in dissociated Drosophila photoreceptors: evidence for feedback by calcium permeating the light-sensitive channels. Proc. R. Soc. Lond. B Biol. Sci. 245: 203-210, 1991.

HAUSEN, K. Monocular and binocular computation of motion in the lobula plate of the fly. Verh. Dtsch. Zool. Ges. 74: 49-70, 1981.

HAUSEN, K. Motion sensitive interneurons in the optomotor system of the fly. I. The horizontal cells: structure and signals. Biol. Cybern. 45: 143156, 1982a.

HAUSEN, K. Motion sensitive interneurons in the optomotor system of the fly. II. The horizontal cells: receptive field organization and response characteristics. Biol. Cybern. 46: 67-79, 1982b.

HAUSEN, K. AND EGELHAAF, M. Neural mechanisms of visual course control in insects. In: Facets of Vision, edited by D. Stavenga and R. Hardie. Berlin: Springer-Verlag, 1989, p. 391-424.

Hausen, K., Wolburg-BuchHolz, K., AND RibI, W. A. The synaptic organization of visual interneurons in the lobula complex of flies. Cell Tissue Res. 208: 371-387, 1980.

HENGSTENBERG, R. Spike responses of "non-spiking" visual interneurone. Nature Lond. 270: 338-340, 1977.

Hengstenberg, R. Common visual response properties of giant vertical cells in the lobula plate of the blowfly Calliphora. J. Comp. Physiol. 149: 179-193, 1982.

Hengstenberg, R., Hausen, K., AND Hengstenberg, B. The number and structure of giant vertical cells (VS) in the lobula plate of the blowfly, Calliphora erythrocephala. J. Comp. Physiol. 149: 163-177, 1982.

JafFe, D. B., Johnston, D., LAsser-Ross, N., Lisman, J. E., MiyaKawa, H., AND Ross, W. N. The spread of Na spikes determnines the pattern of dendritic $\mathrm{Ca}^{2+}$ entry into hippocampal neurons. Nature Lond. 357: 244-246, 1992.

Kasai, H. ANd Petersen, O. H. Spatial dynamics of second messengers: IP3 and cAMP as long-range and associative messengers. Trends Neurosci. 17: 95-102, 1994.

Krapp, H., Hengstenberg, B., ANd Hengstenberg, R. Correspondence of dendritic field structure, receptive field organization, and specific flow patterns in visual interneurons of the blowfly Calliphora. In: Sensory Transduction, edited by N. Elsner and H. Breer. Stuttgart, Germny: Thieme, 1994, p. 453.

LAURENT, G. Voltage-dependent nonlinearities in the membrane of locust nonspiking local interneurons, and their significance for synaptic integration. J. Neurosci. 10: 2268-2280, 1990.

LAURENT, G. A dendritic gain control mechanism in axonless neurons of the locust, Schistocerca americana. J. Physiol. Lond. 470: 45-54, 1993.

Lenting, B. P. M., Mastebroek, H. A. K., ANd ZaAgman, W. H. Saturation in a wide-field, directionally selective movement detection system in fly vision. Vision Res. 24: 1341-1347, 1984.

Lev -Ram, V., Miyakawa, H., Lasser-Ross, N., and Ross, W. N. Calcium transients in cerebellar Purkinje neurons evoked by intracellular stimulation. J. Neurophysiol. 68: 1167-1177, 1992.

LLINÁs, R. R. The intrinsic electrophysiological properties of mammalian neurons: insights into central nervous system function. Science Wash. DC 242: 1654-1664, 1988.

LLINÁs, R. R. AND SUGIMORI, M. Electrophysiological properties of in vitro Purkinje cell somata in mammalian cerebellar slices. J. Physiol. Lond. 305: 171-195, 1980a.

LLINÁs, R. R. AND SUGIMORI, M. Electrophysiological properties of in vitro Purkinje cell dendrites in mammalian cerebellar slices. J. Physiol. Lond. 305: 197-213, 1980b. 
MAdDESS, T. Afterimage-like effects in the motion-sensitive neuron $\mathrm{H} 1$. Proc. R. Soc. Lond. B Biol. Sci. 228: 433-459, 1986.

Maddess, T. and Laughlin, S. B. Adaptation of the motion-sensitive neuron $\mathrm{H} 1$ is generated locally and governed by contrast frequency. Proc. R. Soc. Lond. B Biol. Sci. 225: 251-275, 1985.

MidTGAARD, J. Processing of information from different sources: spatial synaptic integration in the dendrites of vertebrate CNS neurons. Trends Neurosci. 17: 166-173, 1994.

Miyakawa, H., Lev-Ram, V., Lasser-Ross, N., and Ross, W. N. Calcium transients evoked by climbing fiber and parallel fiber synaptic inputs in guinea pig cerebellar Purkinje cells. J. Neurophysiol. 68: $1178-1189,1992$.

Regehr, W. G., Connor, J. A., And Tank, D. W. Optical imaging of calcium accumulation in hippocampal pyramidal cells during synaptic activation. Nature Lond. 341: 533-536, 1989.

REGEHR, W. G. AND TANK, D. W. Postsynaptic NMDA receptor mediated calcium accumulation in hippocampal CA1 pyramidalcell dendrites. $\mathrm{Na}$ ture Lond. 345: 807-810, 1990.

REgeHR, W. G. AND TANK, D. W. Calcium concentration dynamics produced by synaptic activation of CA1 hippocampal pyramidal cells. $J$. Neurosci. 12: 4202-4223, 1992.

REICHARDT, W. Autocorrelation, a principle for the evaluation of sensory information by the central nervous system. In: Sensory Communication, edited by W. A. Rosenblith. New York: M.I.T. Press and London: Wiley, 1961, p. 303-317.

RFICHARDT, W. Evaluation of optical motion information by movement detectors. J. Comp. Physiol. A Sens. Neural Behav. Physiol. 161: 533$547,1987$.

Ross, W. N., Stockbridge, L. L., And Stockbridge, N. L. Regional properties of calcium entry in barnacle neurons determined with arsenazo III and a photodiode array. J. Neurosci. 6: 1148-1159, 1986.

Ross, W. N. AND WERMAN, R. Mapping calcium transients in the dendrites of Purkinje cells from the guinea pig cerebellum in vitro. J. Physiol. Lond. 389: 319-336, 1987.

SANDLER, C. AND KIRSCHFELD, K. Light-induced extracellular calcium and sodium concentration changes in the retina of Calliphora: involvement in the mechanism of light adaptation. J. Comp. Physiol. A Sens. Neural Behav. Physiol. 169: 299-311, 1991.

Sobel, E. C. AND TANK, D. W. In vivo calcium dynamics in a cricket auditory neuron: an example of chemical computation. Science Wash. DC 263: 823-827, 1994.

STRAUSFELD, N. J. AND LEE, J.-K. Neuronal basis for parallel visual processing in the fly. Visual Neurosci. 7: 13-33, 1991.

Swandulla, D., CARBONE, E., AND LuX, H. D. Do calcium channel classifications account for neuronal calcium channel diversity? Trends Neurosci. 14: 46-51, 1991.

Tsien, R. W., Lipscombe, D., Madison, D. V., Bley, K. R., and FoX, A. P. Multiple types of neuronal channels and their selective modulation. Trends Neurosci. 11: 431-438, 1988.

VRANESIC, I. AND KNÖ PFFI, T. Calculation of calcium dynamics from single wavelength fura-2 fluorescence recordings. Pfluegers Arch. 418: 184$189,1991$. 